\title{
Classical Solvability of a Free Boundary Problem for an Incompressible Viscous Fluid with a Surface Density Equation
}

\author{
Yoshiaki Kusaka \\ Department of Mathematics, Faculty of Engineering, Tamagawa University, 6-1-1 Tamagawa-Gakuen, Machida, Tokyo 194-8610, Japan \\ Correspondence should be addressed to Yoshiaki Kusaka; kusaka@eng.tamagawa.ac.jp
}

Received 16 June 2013; Accepted 8 August 2013

Academic Editor: Rodrigo Lopez Pouso

Copyright ( 2013 Yoshiaki Kusaka. This is an open access article distributed under the Creative Commons Attribution License, which permits unrestricted use, distribution, and reproduction in any medium, provided the original work is properly cited.

We investigate a mathematical model introduced by Shikhmurzaev to remove singularities that arise when classical hydrodynamic models are applied to certain physical phenomena. The model is described as a free boundary problem consisting of the NavierStokes equations and a surface mass balance equation. We prove the local-in-time solvability in Hölder spaces.

\section{Introduction}

Let a time-dependent bounded domain $\Omega_{t} \subset \mathbf{R}^{3}$ with the outer boundary $\Gamma_{t} \equiv \partial \Omega_{t}$ be filled with an incompressible viscous fluid, and let $\Gamma_{t}$ represent the interface. In $\Omega_{t}$, we assume that the flow is governed by the Navier-Stokes equations:

$$
\frac{\partial \mathbf{v}}{\partial t}+(\mathbf{v} \cdot \nabla) \mathbf{v}+\nabla p-v \Delta \mathbf{v}=\mathbf{0}, \quad \nabla \cdot \mathbf{v}=0
$$

where $\mathbf{v}$ is the velocity, $p$ is the pressure, and $v$, which is assumed to be a positive constant, is the kinematic viscosity.

On $\Gamma_{t}$, we assume the following equations:

$$
\begin{gathered}
\Pi \mathbf{T}(\mathbf{v}, p) \mathbf{n}=\bar{\nabla} \sigma, \quad \mathbf{n} \cdot \mathbf{T}(\mathbf{v}, p) \mathbf{n}=\sigma H, \\
\frac{D \rho^{s}}{D t}+\rho^{s} \bar{\nabla} \cdot \mathbf{v}^{s}=\rho\left(\mathbf{v}-\mathbf{v}^{s}\right) \cdot \mathbf{n}, \\
\left(\mathbf{v}-\mathbf{v}^{s}\right) \cdot \mathbf{n}=-\frac{\rho_{e}^{s}-\rho^{s}}{\rho \tau}, \quad \Pi\left(\mathbf{v}-\mathbf{v}^{s}\right)=-\chi \bar{\nabla} \sigma, \\
\sigma=\gamma\left(\bar{\rho}-\rho^{s}\right) .
\end{gathered}
$$

Here $\mathbf{v}^{s}$ and $\rho^{s}$ are the velocity and the density of surface layer, respectively. $\mathbf{T}(\mathbf{v}, p)=\nu \mathbf{D}(\mathbf{v})-p I$ is the stress tensor, where $\mathbf{D}(\mathbf{v})=\left(\left(\partial v_{i} / \partial x_{j}\right)+\left(\partial v_{i} / \partial x_{j}\right)\right)_{i, j=1,2,3}$ is the velocity deformation tensor. $H$ is the twice mean curvature of $\Gamma_{t}$ at the point $x$, which is negative if $\Omega_{t}$ is convex in the neighborhood of $x . \mathbf{n}$ is the unit outward normal to $\Gamma_{t}$ at the point $x . \Pi$ is the projection operator onto the tangent plane at the point $x$ on $\Gamma_{t} . D / D t$ denotes the derivative along the trajectory of particle on $\Gamma_{t} . \bar{\nabla}$ is the gradient restricted to the surface. $\rho, \rho_{e}^{s}$, $\tau, \chi, \gamma, \bar{\rho}$ are positive constants; in particular, $\rho$ is the density of the bulk and $\tau$ is the characteristic time scale over which the surface density $\rho^{s}$ relaxes to its equilibrium value $\rho_{e}^{s}$.

Finally, to complete the problem, we give the initial conditions:

$$
\left.\mathbf{v}\right|_{t=0}=\mathbf{v}_{0} \quad \text { on } \bar{\Omega} \equiv \bar{\Omega}_{0},\left.\quad \rho^{s}\right|_{t=0}=\rho_{0} \quad \text { on } \Gamma \equiv \Gamma_{0} .
$$

It is known that singularities arise when the the classical hydrodynamic equations and modeling assumptions are applied to certain physical phenomena. For example, the application of the classical no-slip boundary condition to the spreading of a drop on a plate gives rise to a nonintegrable shear stress, and the application of the classical kinematic condition at the free boundary to the formation of a cusp on a free surface of a viscous fluid leads to an infinite energy dissipation in the fluid (e.g., refer to [1] and the references therein).

To remove the above mentioned singularities, we are required to modify classical boundary conditions by taking into account molecular interaction near interfaces. The molecule in the liquid region which is very close to another phases experiences an asymmetric force due to the presence of another materials. This gives rise to the variation in the 
density in the liquid region near to the adjacent phase, and the surface tension occurs as a result of this variation in density. The thin layer in the liquid region in which the above mentioned density variation occurs is called the surface layer.

Through [1-4], Shikhmurzaev developed a theory to remove the above mentioned singularities by introducing a surface layer which is treated as a separate phase. In this theory, the no-slip condition assumed in classical models for dynamic wetting processes is modified as the Navierslip condition through thermodynamic considerations on the surface layer (refer to $[2,3]$ ). The formation of a free surface cusp associated with fluid flow is also investigated in [4]. In [4], the cusp formation is modeled as an interface disappearance process. In this model, an internal surface stretching from the cusp, which is referred to as "the surfacetension-relaxation tail", is introduced. The above mentioned singularity associated with the modeling of cusp formation arises owing to the absence of viscous stress at the cusp with which the surface tension acting from the liquid surface is balanced. In this model, the surface tension at the cusp can be balanced by shear stresses acting on this tail.

The problem (1)-(6) is a model describing the behavior of an isolated liquid drop in which the interface is modeled as a surface layer based on Shikhmurzaev's theory. The dynamics of the liquid in this layer are governed by (3) which represents conservation of mass. The right-hand side of (3) represents the source consisting of a flow of molecules from the bulk. Equations in (4) are conditions that minimize the rate of entropy production in the surface layer. Equation (5) represents a linearized state equation in the surface layer (refer to [1] for details). In Shikhmurzaev's theory, the surface layer is modeled as a sharp interface as a result of a continuum approximation. Thus, in the above problem, the surface layer is described by the equations given in (3)-(5) defined on a geometric surface, and the behavior of the surface layer is related to (1) in the bulk through the boundary conditions given in (2).

In the present paper, we prove the local-in-time classical solvability of problem (1)-(6). As is mentioned above, this model is important as a basic model to describe the above mentioned physical phenomena; however, as far as the author knows, any rigorous proofs on its solvability have not been given. In the present paper, we consider the case where the mass exchange between the interface and the bulk does not occur. As is seen in Section 2, under such an assumption, we can reformulate our problem as a problem defined in a domain with a fixed known boundary by introducing Lagrangian coordinates, and in Section 3, we construct a unique solution of the reformulated problem in Hölder spaces with the aid of the method of successive approximations.

\section{Reformulation of the Problem}

In this section, we reformulate our problem in Lagrangian coordinates. By Lagrangian coordinates we mean the initial coordinates of the fluid particles. In the case where no exchange of molecules occurs between the surface and the bulk, (4) ${ }^{1}$ is reduced to $\mathbf{v} \cdot \mathbf{n}=\mathbf{v}^{s} \cdot \mathbf{n}$. This relation indicates that the following kinematic condition at the interface is satisfied: the interface consists of the particles located at the interface at the initial time. This circumstance enables us to relate each point $x \in \bar{\Omega}_{t}$ to its initial point $\xi \in \bar{\Omega}$ by relation (10) given below.

Before reformulating our problem, we rewrite (3) as a nonlinear parabolic equation on $\Gamma_{t}$ with the time derivative $\widetilde{D} \rho^{s} / \widetilde{D} t$, where $\widetilde{D} \rho^{s} / \widetilde{D} t$ denotes the derivative along the trajectory of particle on the interface with velocity $\mathbf{v}$. Noting the following relation (e.g., see [5]):

$$
\left(\frac{D \rho^{s}}{D t}\right)_{n}=\frac{D \rho^{s}}{D t}-\mathbf{v}^{s} \cdot \bar{\nabla} \rho^{s}=\frac{\widetilde{D} \rho^{s}}{\widetilde{D} t}-\mathbf{v} \cdot \bar{\nabla} \rho^{s},
$$

where $\left(D \rho^{s} / D t\right)_{n}$ represents the derivative along the trajectory which is normal to the interface, (3) can be written as

$$
\frac{\widetilde{D} \rho^{s}}{\widetilde{D} t}+\rho^{s} \bar{\nabla} \cdot \mathbf{v}^{s}=\left(\mathbf{v}-\mathbf{v}^{s}\right) \cdot \bar{\nabla} \rho^{s} .
$$

Then eliminating $\mathbf{v}^{s}$ from the above equation with the aid of the relation $(4)^{2}$, we obtain the following equation:

$$
\begin{aligned}
\frac{\widetilde{D} \rho^{s}}{\widetilde{D} t}-\chi \gamma \rho_{0}^{s} \bar{\nabla}^{2} \rho^{s}= & -\rho^{s} \bar{\nabla} \cdot \mathbf{v} \\
& +\chi \gamma\left\{\left(\rho^{s}-\rho_{0}^{s}\right) \bar{\nabla}^{2} \rho^{s}+\bar{\nabla} \rho^{s} \cdot \bar{\nabla} \rho^{s}\right\} .
\end{aligned}
$$

Now let us reformulate our problem. The Lagrangian and Eulerian coordinates are related by

$$
x=X_{u}(\xi, t) \equiv \xi+\int_{0}^{t} \mathbf{u}(\xi, \tau) d \tau,
$$

where $\mathbf{u}(\xi, \tau)$ is the velocity at time $t$ of the particle which was located at $\xi$ at $t=0$. By changing the variables from $x$ to $\xi$ by relation (10), problem (1)-(6) is reformulated as the following problem defined in the cylindrical domain $\Omega_{0 T}=\Omega \times(0, T)$ with the lateral boundary $\Gamma_{0 T} \equiv \Gamma \times(0, T)$ :

$$
\begin{gathered}
\frac{\partial \mathbf{u}}{\partial t}-\nu \nabla_{u}^{2} \mathbf{u}+\nabla_{u} q=\mathbf{0}, \quad \nabla_{u} \cdot \mathbf{u}=0 \quad \text { in } \Omega_{0 T}, \\
\Pi \Pi_{u} \mathbf{T}_{u}(\mathbf{u}, q) \mathbf{n}_{u}=\Pi \nabla_{\Gamma_{t}} \theta, \\
\mathbf{n} \cdot \mathbf{T}_{u}(\mathbf{u}, q) \mathbf{n}_{u}=\left.\theta \nabla_{\Gamma_{t}}^{2} X_{u}(\xi, t)\right|_{\xi \in \Gamma} \cdot \mathbf{n}+\mathbf{n} \cdot \nabla_{\Gamma_{t}} \theta, \\
\frac{\partial r^{s}}{\partial t}-\chi \gamma \rho_{0}^{s} \nabla_{\Gamma_{t}}^{2} r^{s} \\
=-r^{s} \nabla_{\Gamma_{t}} \cdot \mathbf{u}+\chi \gamma\left\{\left(r^{s}-\rho_{0}^{s}\right) \nabla_{\Gamma_{t}}^{2} r^{s}+\nabla_{\Gamma_{t}} r^{s} \cdot \nabla_{\Gamma_{t}} r^{s}\right\}, \\
\theta=\gamma\left(\bar{\rho}-r^{s}\right) \quad \text { on } \Gamma_{0 T}, \\
\left.\mathbf{u}\right|_{t=0}=\mathbf{v}_{0} \quad \text { on } \bar{\Omega},\left.\quad r^{s}\right|_{t=0}=\rho_{0}^{s} \quad \text { on } \Gamma .
\end{gathered}
$$

In (11)-(13), $\mathbf{u}, q$ and $r^{s}$ are $\mathbf{v}\left(X_{u}(\xi, t), t\right), p\left(X_{u}(\xi, t), t\right)$, and $\rho^{s}\left(X_{u}(\xi, t), t\right)$, respectively. Consider $\nabla_{u}=\left(\mathcal{F}_{u}^{-1}\right)^{t} \nabla \equiv \mathcal{F}_{u}^{*} \nabla$; here $\mathscr{J}_{u}$ denotes the Jacobian matrix of $X_{u}$, and the notation 
$A^{t}$ means the transpose of the matrix $A . \mathbf{n}$ is the outward unit normal to $\Gamma$ at the point $\xi, \mathbf{n}_{u}=\mathscr{J}_{u}^{*} \mathbf{n} /\left|\mathscr{J}_{u}^{*} \mathbf{n}\right|$, and $\Pi$ and $\Pi_{u}$ are the operators defined by $\Pi \mathbf{f}=\mathbf{f}-(\mathbf{f} \cdot \mathbf{n}) \mathbf{f}$ and $\Pi_{u} \mathbf{f}=\mathbf{f}-\left(\mathbf{f} \cdot \mathbf{n}_{u}\right) \mathbf{f}$, respectively. $\mathbf{T}_{u}(\mathbf{u}, q)$ is the tensor with the elements $v \sum_{k=1}^{3}\left(A_{j k}\left(\partial u_{i} / \partial \xi_{k}\right)+A_{i k}\left(\partial u_{j} / \partial \xi_{k}\right)\right)-p \delta_{i j}$, where $A_{i j}$ is the $(i, j)$-element of $\mathcal{J}_{u}^{*}$, and $\delta_{i j}$ is Kronecker's delta. The operators $\nabla_{\Gamma_{t}} f$ and $\nabla_{\Gamma_{t}} \cdot \mathbf{A}$ are defined by

$$
\begin{aligned}
& \nabla_{\Gamma_{t}} f=\sum_{\alpha, \beta=1,2} g^{\alpha \beta} \frac{\partial f}{\partial s_{\beta}} \frac{\partial X_{u}(s)}{\partial s_{\alpha}}, \\
& \nabla_{\Gamma_{t}} \cdot \mathbf{A}=\frac{1}{\sqrt{g}} \sum_{\alpha=1,2} \frac{\partial}{\partial s_{\alpha}} \sqrt{g} A_{\alpha},
\end{aligned}
$$

where $g=\operatorname{det}\left(g_{\alpha \beta}\right)_{\alpha, \beta=1,2}, g_{\alpha \beta}=\left(\partial X_{u}(s) / \partial s_{\alpha}\right) \cdot\left(\partial X_{u}(s) /\right.$ $\left.\partial s_{\beta}\right), g^{\alpha \beta}$ denote the components of the inverse matrix of $\left(g_{\alpha \beta}\right)_{\alpha, \beta=1,2}, X_{u}(s)=X_{u}(\xi(s), t), s=\left(s_{1}, s_{2}\right)$ denotes the local coordinates on $\Gamma$, and $A_{\alpha}$ denotes the components of the vector $\mathbf{A}$ with respect to the basis $\left(\partial X_{u}(s) / \partial s_{\alpha}\right), \alpha=1,2$. Finally, the operator $\nabla_{\Gamma_{t}}^{2} f$ is defined as

$$
\nabla_{\Gamma_{t}}^{2} f=\nabla_{\Gamma_{t}} \cdot \nabla_{\Gamma_{t}} f=\frac{1}{\sqrt{g}} \sum_{\alpha, \beta=1,2} \frac{\partial}{\partial s_{\alpha}} \sqrt{g} g^{\alpha \beta} \frac{\partial f}{\partial s_{\beta}} .
$$

Note that in derivation of $(12)^{2}$, we have used the formula $H \mathbf{n}=\bar{\nabla}^{2} x \equiv \bar{\nabla}^{2} X_{u}(\xi, t)$. Note also that although $(12)^{1,2}$ are different from the following formulas which are obtained directly from (2):

$$
\begin{gathered}
\Pi_{u} \mathbf{T}_{u}(\mathbf{u}, q) \mathbf{n}_{u}=\nabla_{\Gamma_{t}} \theta, \\
\mathbf{n}_{u} \cdot \mathbf{T}_{u}(\mathbf{u}, q) \mathbf{n}_{u}=\left.\theta \nabla_{\Gamma_{t}}^{2} X_{u}(\xi, t)\right|_{\xi \in \Gamma} \cdot \mathbf{n}_{u},
\end{gathered}
$$

problem (11)-(13) is equivalent to problem (1)-(6) as far as the condition $\mathbf{n} \cdot \mathbf{n}_{u}>0$, which is valid for sufficiently small $t$, is satisfied.

We now introduce some function spaces. Let $D$ be a domain in $\mathbf{R}^{n}$, let $T$ be a positive constant, let $D_{T}$ be a cylindrical domain $D \times(0, T)$, let $l$ be a nonnegative integer, and let $\alpha, \gamma \in(0,1)$.

By $C^{l+\alpha}(D)$, we define the space of functions $f(x), x \in D$, with the norm

$$
\begin{gathered}
|f|_{D}^{(l+\alpha)} \equiv \sum_{|m| \leq l}\left|\partial_{x}^{m} f\right|_{D}+[f]_{D}^{(l+\alpha)}, \quad|f|_{D} \equiv \sup _{x \in D}|f(x)| \\
{[f]_{D}^{(l+\alpha)} \equiv \sum_{|m|=l}\left[\partial_{x}^{m} f\right]_{D}^{(\alpha)} \equiv \sup _{x, y \in D, x \neq y} \sum_{m=|l|} \frac{\left|\partial_{x}^{m} f(x)-\partial_{y}^{m} f(y)\right|}{|x-y|^{\alpha}}} \\
|m|=\sum_{i=1}^{n} m_{i}, \quad \partial_{x}^{m}=\frac{\partial^{|m|}}{\partial_{x_{1}}^{m_{1}} \cdots \partial_{x_{n}}^{m_{n}}},
\end{gathered}
$$

for a multi-index $m=\left(m_{i}\right)\left(m_{i} \geq 0, i=1, \ldots, n\right)$.

By $C^{l+\alpha,((l+\alpha) / 2)}\left(D_{T}\right)$ we denote an anisotropic Hölder space of functions whose norm is defined by

$$
|f|_{D_{T}}^{(l+\alpha,((l+\alpha) / 2))} \equiv \sum_{2 r+|m|=0}^{l}\left|\partial_{t}^{r} \partial_{x}^{m} f\right|_{D_{T}}+[f]_{D_{T}}^{(l+\alpha,((l+\alpha) / 2))}
$$

where

$$
\begin{gathered}
|f|_{D_{T}} \equiv \sup _{(x, t) \in D_{T}}|f(x, t)| \\
{[f]_{D_{T}}^{(l+\alpha,((l+\alpha) / 2))} \equiv \sum_{2 r+|m|=l-1}^{l}\left[\partial_{t}^{r} \partial_{x}^{m} f\right]_{D_{T}}^{(0,((l+\alpha-(2 r+|m|)) / 2))}} \\
+\sum_{2 r+|m|=l}\left[\partial_{t}^{r} \partial_{x}^{m} f\right]_{D_{T}}^{(\alpha, 0)}
\end{gathered}
$$

Here,

$$
\begin{aligned}
& {[f]_{D_{T}}^{(0,(\alpha / 2))} \equiv \sup _{(x, t),\left(x, t^{\prime}\right) \in D_{T}, t \neq t^{\prime}} \frac{\left|f(x, t)-f\left(x, t^{\prime}\right)\right|}{\left|t-t^{\prime}\right|^{(\alpha / 2)}},} \\
& {[f]_{D_{T}^{(\alpha, 0)}}^{(\alpha)} \sup _{(x, t),\left(x^{\prime}, t\right) \in D_{T}, x \neq x^{\prime}} \frac{\left|f(x, t)-f\left(x^{\prime}, t\right)\right|}{\left|x-x^{\prime}\right|^{\alpha}} .}
\end{aligned}
$$

Finally, we introduce the function space $C^{1+\alpha, \gamma}\left(D_{T}\right)$ equipped with the norm

$$
\|f\|_{D_{T}}^{(1+\alpha, \gamma)} \equiv|f|_{D_{T}}+|\nabla f|_{D_{T}}^{(\alpha,(\alpha / 2))}+|f|_{D_{T}}^{(1+\alpha, \gamma)}
$$

where

$$
|f|_{D_{T}}^{(1+\alpha, \gamma)} \equiv \sup _{\tau, t \in(0, T), \tau \neq t} \frac{|f(\cdot, t)-f(\cdot, \tau)|_{D}^{(\gamma)}}{|t-\tau|^{((1+\alpha-\gamma) / 2)}} .
$$

Now, let us state our main result.

Theorem 1. Let $\alpha, \gamma$ be constants satisfying $0<\alpha, \gamma<1$. Assume that

$$
\mathbf{v}_{0} \in C^{2+\alpha}(\bar{\Omega}), \quad \rho_{0}^{s} \in C^{2+\alpha}(\Gamma), \quad \Gamma \in C^{3+\alpha}
$$

Assume that there exist positive constants $\Delta_{1}$ and $\Delta_{2}$ such that $\bar{\rho}-\rho_{0}^{s} \geq \Delta_{1}>0$ and $\rho_{0}^{s} \geq \Delta_{2}>0$ on $\Gamma$. In addition, assume that the following compatibility conditions are satisfied:

$$
\nabla \cdot \mathbf{v}_{0}=\mathbf{0},\left.\quad\left(\nu \Pi \mathbf{D}\left(\mathbf{v}_{0}\right) \mathbf{n}+\gamma \nabla_{\Gamma} \rho_{0}^{s}\right)\right|_{\Gamma}=\mathbf{0},
$$

where $\nabla_{\Gamma}$ is the operator corresponding to $\nabla_{\Gamma_{t}}$ with $t=0$; namely, $\nabla_{\Gamma}$ is given by the formula in (14) with $X_{u}(s)=\xi(s)$.

Then, for a positive constant $T$, problem (11)-(13) has a unique solution $\left(\mathbf{u}, q, r^{s}\right)$ with the following smoothness:

$$
\begin{gathered}
\mathbf{u} \in C^{2+\alpha, 1+(\alpha / 2)}\left(\Omega_{0 T}\right), \\
q \in C^{1+\alpha, \gamma}\left(\Omega_{0 T}\right) \cap C^{1+\alpha,((1+\alpha) / 2)}\left(\Gamma_{0 T}\right), \\
r^{s} \in C^{2+\alpha, 1+(\alpha / 2)}\left(\Gamma_{0 T}\right) .
\end{gathered}
$$

\section{Proof of the Main Result}

In this section, we will prove Theorem 1 . 
We begin with preparing estimates of solutions to some linear problems. For the following problem:

$$
\begin{gathered}
\frac{\partial \mathbf{u}}{\partial t}-v \nabla_{w}^{2} \mathbf{u}+\nabla_{w} q=\mathbf{F}_{1}, \quad \nabla_{w} \cdot \mathbf{u}=F_{2} \quad \text { in } \Omega_{0 T}, \\
v \Pi \Pi_{w} \mathbf{D}_{w}(\mathbf{u}) \mathbf{n}_{w}=\mathbf{F}_{3}, \\
\mathbf{n} \cdot \mathbf{T}_{w}(\mathbf{u}, q) \mathbf{n}_{w}-\bar{\Theta} \mathbf{n} \cdot \nabla_{\widetilde{\Gamma}_{t}}^{2} \int_{0}^{t} \mathbf{u} d \tau=b+\int_{0}^{t} B d \tau \quad \text { on } \Gamma_{0 T}, \\
\left.\mathbf{u}\right|_{t=0}=\mathbf{v}_{0} \quad \text { on } \bar{\Omega},
\end{gathered}
$$

the following result is given in [6]. In (26), $\nabla_{w}, \Pi_{w}, \mathbf{T}_{w}(\mathbf{u}, q)$ $\left(=\nu \mathbf{D}_{w}(\mathbf{u})-q I\right), \mathbf{n}_{w}$ are defined for a given vector $\mathbf{w}$ in the same manner as $\nabla_{u}, \Pi_{u}, \mathbf{T}_{u}(\mathbf{u}, q), \mathbf{n}_{u}$ are defined, and $\nabla_{\widetilde{\Gamma}_{t}}$ is defined by (14) with $X_{u}=X_{w}$.

Theorem 2. Let $T>0$, and let $\alpha, \gamma$ be positive constants satisfying $0<\alpha, \gamma<1$. Assume that

$$
\begin{gathered}
\Gamma \in C^{2+\alpha}, \quad \mathbf{v}_{0} \in C^{2+\alpha}(\bar{\Omega}), \\
\mathbf{F}_{1} \in C^{\alpha,(\alpha / 2)}\left(\Omega_{0 T}\right), \quad F_{2} \in C^{1+\alpha,((1+\alpha) / 2)}\left(\Omega_{0 T}\right), \\
\mathbf{F}_{3} \in C^{1+\alpha,((1+\alpha) / 2)}\left(\Gamma_{0 T}\right), \quad b \in C^{1+\alpha,((1+\alpha) / 2)}\left(\Gamma_{0 T}\right), \\
B \in C^{\alpha,(\alpha / 2)}\left(\Gamma_{0 T}\right), \quad \bar{\Theta} \in C^{\alpha}(\Gamma) .
\end{gathered}
$$

Assume that there exists a positive constant $\Delta_{1}$ such that $\bar{\Theta} \geq \Delta_{1}>0$ on $\Gamma$. Assume that the following compatibility conditions are satisfied:

$$
\nabla \cdot \mathbf{v}_{0}=F_{2}(\xi, 0),\left.\quad \nu \Pi \mathbf{D}\left(\mathbf{v}_{0}\right) \mathbf{n}\right|_{\Gamma}=\mathbf{F}_{3}(\xi, 0) .
$$

Assume that there exist functions $\mathbf{h} \in C^{\alpha,(\alpha / 2)}\left(\Omega_{0 T}\right), \mathbf{H}_{k}, k=$ $1,2,3$, with a finite norm $\left|\mathbf{H}_{k}\right|_{\Omega_{0 T}}^{(1+\alpha, \gamma)}$ satisfying the relation

$$
\frac{\partial F_{2}}{\partial t}-\nabla_{w} \cdot \mathbf{F}_{1}=\nabla \cdot \mathbf{h}, \quad \mathbf{h}=\sum_{k=1}^{3} \partial_{\xi_{k}} \mathbf{H}_{k}
$$

in the sense of distribution. Furthermore, assume that $\mathbf{w} \in$ $C^{2+\alpha, 1+(\alpha / 2)}\left(\Omega_{0 T}\right)$ satisfies the inequality

$$
\left(T+T^{1 / 2}\right)|\mathbf{w}|_{\Omega_{0 T}}^{(2+\alpha, 1+(\alpha / 2))}+T^{((1-\alpha+\gamma) / 2)}\left|\partial_{\xi} \mathbf{w}\right|_{\Omega_{0 T}} \leq \delta,
$$

Then problem (26) has a unique solution (u, q) satisfying the following inequality:

$$
\begin{aligned}
|\mathbf{u}|_{\Omega_{0 T}}^{(2+\alpha, 1+(\alpha / 2))}+ & \|q\|_{\Omega_{0 T}}^{(1+\alpha, \gamma)}+|q|_{\Gamma_{0 T}}^{(1+\alpha,((1+\alpha) / 2))} \\
\leq \bar{C}_{1}(T) & \left\{\left|\mathbf{F}_{1}\right|_{\Omega_{0 T}}^{(\alpha,(\alpha / 2))}+\left|F_{2}\right|_{\Omega_{0 T}}^{(1+\alpha,((1+\alpha) / 2))}\right. \\
& +\left|\mathbf{F}_{3}\right|_{\Gamma_{0 T}}^{(1+\alpha,((1+\alpha) / 2))}+|b|_{\Gamma_{0 T}}^{(1+\alpha,((1+\alpha) / 2))} \\
& +|B|_{\Gamma_{0 T}}^{(\alpha,(\alpha / 2))}+\left|\mathbf{v}_{0}\right|_{\Omega}^{(2+\alpha)}+|\mathbf{h}|_{\Omega_{0 T}}^{(\alpha,(\alpha / 2))} \\
& \left.+\sum_{k=1}^{3}\left|\mathbf{H}_{k}\right|_{\Omega_{0 T}}^{(1+\alpha, \gamma)}+P_{T}(\mathbf{w})\left(\left|\mathbf{v}_{0}\right|_{\Omega}^{(1)}+|b(\cdot, 0)|_{\Gamma}\right)\right\},
\end{aligned}
$$

where $P_{T}(\mathbf{w})=T^{((1-\alpha) / 2)}|\mathbf{w}|_{\Omega_{0 T}}^{(1,0)}+\left|\partial_{\xi} \mathbf{w}\right|_{\Omega_{0 T}}^{(\alpha,(\alpha / 2))}+$ $\left[\partial_{\xi} \mathbf{w}\right]_{\Omega_{0 T}}^{(0,((1+\alpha-\gamma) / 2))}$ and $\bar{C}_{1}(T)$ is a nondecreasing function of $T$.

For the following problem:

$$
\begin{gathered}
\frac{\partial r^{s}}{\partial t}-\chi \gamma \rho_{0}^{s} \nabla_{\widetilde{\Gamma}_{t}}^{2} r^{s}=G \quad \text { on } \Gamma_{0 T}, \\
\left.r^{s}\right|_{t=0}=\rho_{0}^{s} \quad \text { on } \Gamma,
\end{gathered}
$$

we have the following theorem. The assertion of the theorem immediately follows from the Hölder estimates for linear parabolic equations (e.g., see [7]).

Theorem 3. Let $T>0$, and let $\alpha$ be a positive constant satisfying $0<\alpha<1$. Assume that

$$
\Gamma \in C^{2+\alpha}, \quad \rho_{0}^{s} \in C^{2+\alpha}(\Gamma), \quad G \in C^{\alpha,(\alpha / 2)}\left(\Gamma_{0 T}\right) .
$$

Assume that there exists a positive constant $\Delta_{2}$ such that $\rho_{0}^{s} \geq$ $\Delta_{2}>0$ on $\Gamma$. Further assume the same assumptions for $\mathbf{w}$ stated in Theorem 2.

Then, problem (32) has a unique solution $r^{\text {s }}$ satisfying the following inequality:

$$
\left|r^{s}\right|_{\Gamma_{0 T}}^{(2+\alpha, 1+(\alpha / 2))} \leq \bar{C}_{2}(T)\left(|G|_{\Gamma_{0 T}}^{(\alpha,(\alpha / 2))}+\left|\rho_{0}^{s}\right|_{\Gamma}^{(2+\alpha)}\right),
$$


Combining the above results, we can easily obtain Theorem 4 given below for the following problem:

$$
\begin{gathered}
\frac{\partial \mathbf{u}}{\partial t}-\nu \nabla_{w}^{2} \mathbf{u}+\nabla_{w} q=\mathbf{F}_{1}, \quad \nabla_{w} \cdot \mathbf{u}=F_{2} \quad \text { in } \Omega_{0 T}, \\
\nu \Pi \Pi_{w} \mathbf{D}_{w}(\mathbf{u}) \mathbf{n}_{w}+\gamma \Pi \nabla_{\widetilde{\Gamma}_{t}} r^{s}=\mathbf{F}_{3}, \\
\mathbf{n} \cdot \mathbf{T}_{w}(\mathbf{u}, q) \mathbf{n}_{w}-\bar{\Theta} \mathbf{n} \cdot \nabla_{\widetilde{\Gamma}_{t}}^{2} \int_{0}^{t} \mathbf{u} d \tau+\gamma \mathbf{n} \cdot \nabla_{\widetilde{\Gamma}_{t}} r^{s} \\
=b+\int_{0}^{t} B d \tau \quad \text { on } \Gamma_{0 T}, \\
\left.\mathbf{u}\right|_{t=0}=\mathbf{v}_{0} \quad \text { on } \bar{\Omega}, \\
\frac{\partial r^{s}}{\partial t}-\chi \gamma \rho_{0}^{s} \nabla_{\widetilde{\Gamma}_{t}}^{2} r^{s}=G \quad \text { on } \Gamma_{0 T}, \\
\left.r^{s}\right|_{t=0}=\rho_{0}^{s} \quad \text { on } \Gamma .
\end{gathered}
$$

The estimate given in the theorem will be essentially used in the later argument to prove Theorem 1.

Theorem 4. Under the same assumptions given in Theorem 2 where only compatibility condition is replaced by

$\nabla \cdot \mathbf{v}_{0}=F_{2}(\xi, 0),\left.\quad\left(\nu \Pi \mathbf{D}\left(\mathbf{v}_{0}\right) \mathbf{n}+\gamma \Pi \nabla_{\Gamma} \rho_{0}^{s}\right)\right|_{\Gamma}=\mathbf{F}_{3}(\xi, 0)$

and Theorem 3, problem (35) has a unique solution (u, $q, r^{s}$ ) satisfying the following inequality:

$$
\begin{aligned}
|\mathbf{u}|_{\Omega_{0 T}}^{(2+\alpha, 1+(\alpha / 2))}+ & \|\left. q\right|_{\Omega_{0 T}} ^{(1+\alpha, \gamma)}+|q|_{\Gamma_{0 T}}^{(1+\alpha,((1+\alpha) / 2))}+\left|r^{s}\right|_{\Gamma_{0 T}}^{(2+\alpha, 1+(\alpha / 2))} \\
\leq \bar{C}_{3}(T) & \left\{\left|\mathbf{F}_{1}\right|_{\Omega_{0 T}}^{(\alpha,(\alpha / 2))}+\left|F_{2}\right|_{\Omega_{0 T}}^{(1+\alpha,((1+\alpha) / 2))}\right. \\
& +\left|\mathbf{F}_{3}\right|_{\Gamma_{0 T}}^{(1+\alpha,((1+\alpha) / 2))}+|b|_{\Gamma_{0 T}}^{(1+\alpha,((1+\alpha) / 2))} \\
& +|B|_{\Gamma_{0 T}}^{(\alpha,(\alpha / 2))}+|\mathbf{h}|_{\Omega_{0 T}}^{(\alpha,(\alpha / 2))}+\sum_{k=1}^{3}\left|\mathbf{H}_{k}\right|_{\Omega_{0 T}}^{(1+\alpha, \gamma)} \\
& +\left|\mathbf{v}_{0}\right|_{\Omega}^{(2+\alpha)}+|G|_{\Gamma_{0 T}}^{(\alpha,(\alpha / 2))}+\left|\rho_{0}^{s}\right|_{\Gamma}^{(2+\alpha)} \\
& \left.+P_{T}(\mathbf{w})\left(\left|\mathbf{v}_{0}\right|_{\Omega}^{(1)}+|b(\cdot, 0)|_{\Gamma}\right)\right\}
\end{aligned}
$$

where $\bar{C}_{3}(T)$ is a nondecreasing function of $T$. later.

In addition, we prepare estimates for $\mathscr{J}_{u}^{*}$, which are used

Lemma 5. Let $\mathscr{F}_{u}$ and $\mathscr{J}_{u^{\prime}}$ be the Jacobian matrices of the mappings $X_{u}$ and $X_{u^{\prime}}$, respectively. Let us assume that $\mathbf{u}$ and $\mathbf{u}^{\prime}$ satisfy condition (30) for sufficiently small $\delta>0$. Then, the following inequalities hold:

$$
\left|\mathscr{F}_{u}^{*}\right|_{\Omega_{0 T}}^{(1+\alpha,((1+\alpha) / 2))},\left|\mathscr{F}_{u}^{*}\right|_{\Omega_{0 T}}^{(1+\alpha, \gamma)} \leq C,
$$

where $C$ is a positive constant independent of $\delta$, and

$$
\begin{aligned}
& \quad\left|\mathscr{J}_{u}^{*}-\mathscr{J}_{u^{\prime}}^{*}\right|_{\Omega_{0 T}}^{(1+\alpha,((1+\alpha) / 2))},\left|\mathscr{J}_{u}^{*}-\mathscr{J}_{u^{\prime}}^{*}\right|_{\Omega_{0 T}}^{(1+\alpha, \gamma)} \\
& \quad \leq \epsilon\left|\mathbf{u}-\mathbf{u}^{\prime}\right|_{\Omega_{0 T}}^{(2+\alpha, 1+(\alpha / 2))}+C(\epsilon) \int_{0}^{T}\left|\mathbf{u}-\mathbf{u}^{\prime}\right|_{\Omega_{0 \tau}}^{(2+\alpha, 1+(\alpha / 2))} d \tau,
\end{aligned}
$$

for arbitrary $0<\epsilon<1$, where $C(\epsilon)$ is a positive constant depending only on $\epsilon$.

Proof. In the following proof, $c_{1}, c_{2}$, and $c_{3}$ are positive constants independent of $\delta$ and $\epsilon$. Let $a_{i j}$ be the $(i, j)$ component of $\mathscr{J}_{u}$.

Then, we have

$$
\delta_{i j}-\left|\int_{0}^{t} \partial_{\xi_{j}} u_{i} d \tau\right| \leq a_{i j} \leq \delta_{i j}+\left|\int_{0}^{t} \partial_{\xi_{j}} u_{i} d \tau\right|,
$$

where $\delta_{i j}$ denotes Kronecker's delta. Then, using the inequality

$$
\left|\int_{0}^{t} \partial_{\xi_{j}} u_{i} d \tau\right|_{\Omega_{0 T}} \leq T|\mathbf{u}|_{\Omega_{0 T}}^{(1,0)} \leq \delta,
$$

from (40), we have

$$
\delta_{i j}-\delta \leq\left|a_{i j}\right|_{\Omega_{0 T}} \leq \delta_{i j}+\delta
$$

This inequality implies that $\operatorname{det} \mathscr{J}_{u}>0$ holds for sufficiently small $\delta>0$.

Now, let $a_{i j}^{\prime}$ be the $(i, j)$-components of $\mathscr{J}_{u^{\prime}}$, and let $A_{i j}$ and $A_{i j}^{\prime}$ be the cofactors of $a_{i j}$ and $a_{i j}^{\prime}$, respectively.

Then from the inequalities

$$
\left|a_{i j}\right|_{\Omega_{0 T}}^{(1+\alpha,((1+\alpha) / 2))},\left|a_{i j}^{\prime}\right|_{\Omega_{0 T}}^{(1+\alpha,((1+\alpha) / 2))} \leq c_{1}(1+\delta),
$$

we have

$$
\left|A_{i j}\right|_{\Omega_{0 T}}^{(1+\alpha,((1+\alpha) / 2))},\left|A_{i j}\right|_{\Omega_{0 T}}^{(1+\alpha, \gamma)} \leq c_{2} .
$$

On the other hand, from the inequality

$$
\begin{aligned}
\mid a_{i j}- & \left.a_{i j}^{\prime}\right|_{\Omega_{0 T}} ^{(1+\alpha,((1+\alpha) / 2))} \\
\leq & \epsilon\left|\mathbf{u}-\mathbf{u}^{\prime}\right|_{\Omega_{0 T}}^{(2+\alpha, 1+(\alpha / 2))} \\
& +c_{3}\left(1+\epsilon^{-((1+\alpha) /(2-\alpha))}\right) \int_{0}^{T}\left|\mathbf{u}-\mathbf{u}^{\prime}\right|_{\Omega_{0 \tau}}^{(2+\alpha, 1+(\alpha / 2))} d \tau,
\end{aligned}
$$


which are obtained with the aid of the following inequality which holds for arbitrary $0<\epsilon<1$ :

$$
\begin{aligned}
& \left|\int_{0}^{t} f(\xi, \tau) d \tau\right|_{\Omega_{0 T}}^{(1+\alpha,((1+\alpha) / 2))} \\
& \leq \int_{0}^{T}|f|_{\Omega_{0 \tau}}^{(1+\alpha, 0)} d \tau \\
& \quad+\sup _{\left|t^{\prime}-t^{\prime \prime}\right|<\epsilon^{(2 /(1-\alpha))}}\left(\left|t^{\prime}-t^{\prime \prime}\right|^{-((1+\alpha) / 2)} \int_{t^{\prime \prime}}^{t^{\prime}}|f(\xi, \tau)|_{\Omega} d \tau\right. \\
& \left.\quad+\left|t^{\prime}-t^{\prime \prime}\right|^{-(\alpha / 2)} \int_{t^{\prime \prime}}^{t^{\prime}}\left|\partial_{\xi} f(\xi, \tau)\right|_{\Omega} d \tau\right) \\
& \quad+\sup _{\epsilon^{(2 /(1-\alpha))}<\left|t^{\prime}-t^{\prime \prime}\right| \leq T}\left(\left|t^{\prime}-t^{\prime \prime}\right|^{-((1+\alpha) / 2)} \int_{t^{\prime \prime}}^{t^{\prime}}|f(\xi, \tau)|_{\Omega} d \tau\right. \\
& \left.\quad+\left|t^{\prime}-t^{\prime \prime}\right|^{-(\alpha / 2)} \int_{t^{\prime \prime}}^{t^{\prime}}\left|\partial_{\xi} f(\xi, \tau)\right|_{\Omega} d \tau\right) \\
& \leq \epsilon|f|_{\Omega_{0 T}(1+\alpha,((1+\alpha) / 2))} \\
& +c_{3}\left(1+\epsilon^{-((1+\alpha) /(1-\alpha))}\right) \int_{0}^{T}|f|_{\Omega_{0 \tau}}^{(1+\alpha,((1+\alpha) / 2))} d \tau,
\end{aligned}
$$

we have

$$
\begin{aligned}
& \left|A_{i j}-A_{i j}^{\prime}\right|_{\Omega_{0 T}}^{(1+\alpha,((1+\alpha) / 2))},\left|A_{i j}-A_{i j}^{\prime}\right|_{\Omega_{0 T}}^{(1+\alpha, \gamma)} \\
& \quad \leq \epsilon\left|\mathbf{u}-\mathbf{u}^{\prime}\right|_{\Omega_{0 T}}^{(2+\alpha, 1+(\alpha / 2))}+C_{1}(\epsilon) \int_{0}^{T}\left|\mathbf{u}-\mathbf{u}^{\prime}\right|_{\Omega_{0 \tau}}^{(2+\alpha, 1+(\alpha / 2))} d \tau,
\end{aligned}
$$

where $C_{1}(\epsilon)$ is a positive constant depending only on $\epsilon$. From (44) and (47), estimates (38) and (39) immediately follow. Thus, the proof is completed.

Now, let us prove Theorem 1 by the method of successive approximations. We take $\left(\mathbf{u}_{0}, q_{0}, r_{0}^{s}\right)=(\mathbf{0}, 0,0)$, and, for the known $n$th approximation, we define the $(n+1)$ th approximation by the solutions of the following problem:

$$
\begin{gathered}
\frac{\partial \mathbf{u}_{n+1}}{\partial t}-\nu \nabla_{u_{n}}^{2} \mathbf{u}_{n+1}+\nabla_{u_{n}} q_{n+1}=\mathbf{0}, \\
\nabla_{u_{n}} \cdot \mathbf{u}_{n+1}=0 \quad \text { in } \Omega_{0 T}, \\
\nu \Pi \Pi_{u_{n}} \mathbf{D}_{u_{n}}\left(\mathbf{u}_{n+1}\right) \mathbf{n}_{u_{n}}+\gamma \Pi \nabla_{\Gamma_{n, t}} r_{n+1}^{s}=\mathbf{0}, \\
\mathbf{n} \cdot \mathbf{T}_{u_{n}}\left(\mathbf{u}_{n+1}, q_{n+1}\right) \mathbf{n}_{u_{n}}-\bar{\Theta} \mathbf{n} \cdot \nabla_{\Gamma_{n, t}}^{2} \int_{0}^{t} \mathbf{u}_{n+1} d \tau \\
+\gamma \mathbf{n} \cdot \nabla_{\Gamma_{n, t}} r_{n+1}^{s} \\
=b\left(\mathbf{u}_{n}, r_{n}^{s}\right)+\int_{0}^{t} B\left(\mathbf{u}_{n}, r_{n}^{s}\right) d \tau \quad \text { on } \Gamma_{0 T},
\end{gathered}
$$

$$
\begin{gathered}
\left.\mathbf{u}_{n+1}\right|_{t=0}=\mathbf{v}_{0} \quad \text { on } \bar{\Omega}, \\
\frac{\partial r_{n+1}^{s}}{\partial t}-\chi \gamma \rho_{0}^{s} \nabla_{\Gamma_{n, t}}^{2} r_{n+1}^{s}=G\left(r_{n}^{s}, \mathbf{u}_{n}\right) \quad \text { on } \Gamma_{0 T}, \\
\left.r_{n+1}^{s}\right|_{t=0}=\rho_{0}^{s} \quad \text { on } \Gamma,
\end{gathered}
$$

where

$$
\begin{aligned}
b\left(\mathbf{u}_{n}, r_{n}^{s}\right)= & \theta_{n}\left(H_{0}+\mathbf{n} \cdot \int_{0}^{t}\left(\nabla_{\Gamma_{n, \tau}}^{2}\right)_{\tau} \xi d \tau\right), \\
H_{0}=\mathbf{n} \cdot \nabla_{\Gamma}^{2} \xi & \\
B\left(\mathbf{u}_{n}, r_{n}^{s}\right)= & \left\{\frac{\partial}{\partial \tau}\left(\theta_{n}-\bar{\Theta}\right)\right\} \mathbf{n} \cdot \nabla_{\Gamma_{n, \tau}}^{2} \int_{0}^{\tau} \mathbf{u}_{n}(\xi, s) d s \\
& +\left(\theta_{n}-\bar{\Theta}\right) \mathbf{n} \cdot\left(\nabla_{\Gamma_{n, \tau}}^{2}\right)_{\tau} \int_{0}^{\tau} \mathbf{u}_{n}(\xi, s) d s \\
& +\left(\theta_{n}-\bar{\Theta}\right) \mathbf{n} \cdot \nabla_{\Gamma_{n, \tau}}^{2} \mathbf{u}_{n}, \\
\theta_{n}= & \left(\bar{\rho}-r_{n}^{s}\right), \\
G\left(\mathbf{u}_{n}, r_{n}^{s}\right) \equiv & -r_{n}^{s} \nabla_{\Gamma_{n, t}} \cdot \mathbf{u}_{n} \\
& +\chi \gamma\left\{\left(r_{n}^{s}-\rho_{0}^{s}\right) \nabla_{\Gamma_{n, t}}^{2} r_{n}^{s}+\nabla_{\Gamma_{n, t}} r_{n}^{s} \cdot \nabla_{\Gamma_{n, t}} r_{n}^{s}\right\} .
\end{aligned}
$$

In the above formulas, $\nabla_{\Gamma_{n+}}$ is the operator corresponding to $\nabla_{\Gamma_{t}}$ with $X_{u}=X_{u_{n}}$, and $\left(\nabla_{\Gamma_{n, \tau}}^{2}\right)_{\tau}$ denotes the operator obtained by differentiating the coefficients of $\nabla_{\Gamma_{n, \tau}}^{2}$ with respect to $\tau$.

Now, let us verify that all terms of the sequence $\left\{\left(\mathbf{u}_{n}, q_{n}, r_{n}^{s}\right)\right\}$ are defined on some time interval independent of $n$. We begin with the following lemma.

Lemma 6. Let $T_{n}$ be a constant satisfying $0<T_{n} \leq 1$. Then there exist positive constants $\delta$ and $\beta$ such that if $\mathbf{u}_{n}$ and $r_{n}^{s}$ satisfy the following conditions:

$$
\begin{gathered}
\left(T_{n}+T_{n}^{1 / 2}\right)\left|\mathbf{u}_{n}\right|_{\Omega_{0 T_{n}}}^{(2+\alpha, 1+(\alpha / 2))}+T_{n}^{((1-\alpha+\gamma) / 2)}\left|\partial_{\xi} \mathbf{u}_{n}\right|_{\Omega_{0 T_{n}}} \leq \delta, \\
\left(T_{n}+T_{n}^{\beta}\right)\left(\left|\mathbf{u}_{n}\right|_{\Omega_{0 T_{n}}}^{(2+\alpha, 1+(\alpha / 2))}+\left|r_{n}^{s}\right|_{\Gamma_{0 T_{n}}}^{(2+\alpha, 1+(\alpha / 2))}\right) \leq \delta,
\end{gathered}
$$

then the following inequality holds for a positive constant $\bar{C}$ independent of $\mathbf{u}_{n}, r_{n}^{s}$ and $T_{n}$ :

$$
\begin{gathered}
\left|b\left(\mathbf{u}_{n}, r_{n}^{s}\right)\right|_{\Gamma_{0 T_{n}}}^{(1+\alpha,((1+\alpha) / 2))}+\left|B\left(\mathbf{u}_{n}, r_{n}^{s}\right)\right|_{\Gamma_{0 T_{n}}}^{(\alpha,(\alpha / 2))} \\
+\left|G\left(\mathbf{u}_{n}, r_{n}^{s}\right)\right|_{\Gamma_{0 T_{n}}}^{(\alpha,(\alpha / 2))}+P_{T_{n}}\left(\mathbf{u}_{n}\right) \leq \bar{C} .
\end{gathered}
$$

Proof. In the proof, $c_{1}, \ldots, c_{9}$ are positive constants independent of $\mathbf{u}_{n}, r_{n}^{s}$, and $T_{n}$. 
Choosing $T_{n}^{((1-\alpha) / 2 \alpha)}$ as $\epsilon$ in the following interpolation inequality:

$$
\begin{aligned}
& \left|\theta_{n}-\left(\left.\theta_{n}\right|_{t=0}\right)\right|_{\Gamma_{0 T_{n}}}^{(1+\alpha,((1+\alpha) / 2))} \\
& \quad \leq \epsilon\left|r_{n}^{s}-\rho_{0}^{s}\right|_{\Gamma_{0 T_{n}}}^{(2,1)}+c_{1} \epsilon^{-(\alpha /(1-\alpha))}\left|r_{n}^{s}-\rho_{0}^{s}\right|_{\Gamma_{0 T_{n}}} \\
& \quad \leq \epsilon\left|r_{n}^{s}-\rho_{0}^{s}\right|_{\Gamma_{0 T_{n}}}^{(2,1)}+c_{1} \epsilon^{-(\alpha /(1-\alpha))} \int_{0}^{T_{n}}\left|\partial_{\tau}\left(r_{n}^{s}-\rho_{0}^{s}\right)\right|_{\Gamma_{0 \tau}} d \tau,
\end{aligned}
$$

we have the following estimate:

$$
\begin{aligned}
& \left|\theta_{n}\right|_{\Gamma_{0 T_{n}}}^{(1+\alpha,((1+\alpha) / 2))} \\
& \quad \leq c_{2}\left(\left|\rho_{0}^{s}\right|_{\Gamma}^{(1+\alpha)}+\left|\theta_{n}-\left(\left.\theta_{n}\right|_{t=0}\right)\right|_{\Gamma_{0 T_{n}}}^{(1+\alpha,((1+\alpha) / 2))}\right) \\
& \quad \leq c_{3}\left\{\left|\rho_{0}^{s}\right|_{\Gamma}^{(1+\alpha)}+\left(T_{n}^{((1-\alpha) / 2 \alpha)}+T_{n}^{(1 / 2)}\right)\left|r_{n}^{s}-\rho_{0}^{s}\right|_{\Gamma_{0 T_{n}}}^{(2,1)}\right\} \\
& \quad \leq c_{4}(1+\delta) .
\end{aligned}
$$

With the aid of the above estimate, we can easily obtain the desired estimate for $b\left(\mathbf{u}_{n}, r_{n}^{s}\right)$.

$B\left(\mathbf{u}_{n}, r_{n}^{s}\right)$ is estimated as follows. With the aid of the inequality

$$
\begin{aligned}
& \left|\int_{0}^{t} \partial_{\xi}^{2} f(\xi, \tau) d \tau\right|_{\Gamma_{0 T}}^{(\alpha,(\alpha / 2))} \\
& \quad \leq \mathcal{c}_{5}\left(T+T^{1-(\alpha / 2)}\right)|f|_{\Gamma_{0 T}}^{(2+\alpha, 1+(\alpha / 2))}
\end{aligned}
$$

we have

$$
\begin{aligned}
& \mid\left\{\frac{\partial}{\partial t}\left(\theta_{n}-\bar{\Theta}\right)\right\} \mathbf{n} \cdot \nabla_{\Gamma_{n, t}}^{2} \int_{0}^{t} \mathbf{u}_{n}(\xi, \tau) d \tau \\
& \quad+\left.\left(\theta_{n}-\bar{\Theta}\right) \mathbf{n} \cdot\left(\nabla_{\Gamma_{n, t}}^{2}\right) \int_{t}^{t} \int_{0}^{t} \mathbf{u}_{n}(\xi, \tau) d \tau\right|_{\Gamma_{0 T_{n}}} ^{(\alpha,(\alpha / 2))} \\
& \quad \leq c_{6}\left(T_{n}+T_{n}^{1-(\alpha / 2)}\right)\left|r_{n}^{s}-\rho_{0}^{s}\right|_{\Gamma_{0 T_{n}}}^{(2+\alpha, 1+(\alpha / 2))}\left|\mathbf{u}_{n}\right|_{\Gamma_{0 T_{n}}}^{(2+\alpha, 1+(\alpha / 2))} \\
& \quad \leq c_{7}\left(1+\delta^{2}\right) .
\end{aligned}
$$

On the other hand, with the aid of

$$
\begin{aligned}
\left|\theta_{n}-\bar{\Theta}\right|_{\Gamma_{0 T_{n}}}^{(\alpha,(\alpha / 2))} & \leq \int_{0}^{T_{n}}\left|\partial_{\tau}\left(\theta_{n}-\bar{\Theta}\right)\right|_{\Gamma_{0 \tau}}^{(\alpha,(\alpha / 2))} d \tau \\
& \leq T_{n}\left|r_{n}^{s}-\rho_{0}^{s}\right|_{\Gamma_{0 T_{n}}}^{(2+\alpha, 1+(\alpha / 2))},
\end{aligned}
$$

we have

$$
\begin{aligned}
& \left|\left(\theta_{n}-\bar{\Theta}\right) \mathbf{n} \cdot \nabla_{\Gamma_{n, t}}^{2} \mathbf{u}_{n}\right|_{\Gamma_{0 T_{n}}}^{(\alpha,(\alpha / 2))} \\
& \quad \leq c_{8} T_{n}\left|r_{n}^{s}-\rho_{0}^{s}\right|_{\Gamma_{0 T_{n}}}^{(2+\alpha, 1+(\alpha / 2))}\left|\mathbf{u}_{n}\right|_{\Gamma_{0 T_{n}}}^{(2+\alpha, 1+(\alpha / 2))} \\
& \quad \leq c_{9}\left(1+\delta^{2}\right)
\end{aligned}
$$

From (55) and (57), we have the desired estimate for $B\left(\mathbf{u}_{n}, r_{s}^{n}\right)$. $G\left(\mathbf{u}_{n}, r_{n}^{s}\right)$ and $P_{T_{n}}\left(\mathbf{u}_{n}\right)$ are estimated in a similar manner. Thus, we have proved the lemma.

From this lemma, if $\mathbf{u}_{n}$ and $r_{n}^{s}$ satisfy conditions (50), by applying Theorem 4 to problem (3) we have the following estimate of $\mathbf{u}_{n+1}$ and $r_{n+1}^{s}$ :

$$
\left|\mathbf{u}_{n+1}\right|_{\Omega_{0 T_{n}}}^{(2+\alpha, 1+(\alpha / 2))}+\left|r_{n+1}^{s}\right|_{\Gamma_{0 T_{n}}}^{(2+\alpha, 1+(\alpha / 2))} \leq \bar{C}_{3}(1) \bar{C},
$$

where $\bar{C}_{3}(\cdot)$ is the function given in Theorem 4 .

Now, let us take $T$ satisfying the following conditions:

$$
0<T \leq 1, \quad \bar{C}_{3}(1) \bar{C}\left(T+T^{\beta}\right) \leq \delta .
$$

Here $\beta$ is assumed to be chosen, so that $\beta<1 / 2$ and $\beta<$ $(1-\alpha+\gamma) / 2$. Since $\left(\mathbf{u}_{0}, r_{0}^{s}\right)=(\mathbf{0}, 0)$, the zeroth approximation $\left(\mathbf{u}_{0}, r_{0}^{s}\right)$ obviously satisfies conditions (50) for the above $T$. Hence, from (58), $\left(\mathbf{u}_{1}, r_{1}^{s}\right)$ satisfies

$$
\left|\mathbf{u}_{1}\right|_{\Omega_{0 T}}^{(2+\alpha, 1+(\alpha / 2))}+\left|r_{1}^{s}\right|_{\Gamma_{0 T}}^{(2+\alpha, 1+(\alpha / 2))} \leq \bar{C}_{3}(1) \bar{C} .
$$

From (59), this inequality indicates that $\left(\mathbf{u}_{1}, r_{1}^{s}\right)$ satisfies conditions (50), and hence we can obtain the same estimate as (60) for $\left(\mathbf{u}_{2}, r_{2}^{s}\right)$. Thus, repeating this argument, we can construct a sequence $\left\{\left(\mathbf{u}_{n}, q_{n}, r_{n}^{s}\right)\right\}$ such that each term is defined on $(0, T)$.

Let us proceed to the proof of the convergence of the sequence $\left\{\left(\mathbf{u}_{n}, q_{n}, r_{n}^{s}\right)\right\}$. In the following argument, $C_{1}, \ldots, C_{10}$ denote positive constants independent of $n$ and $C(\epsilon)$ represents various positive constants depending only on $\epsilon$.

Let us set

$$
\begin{aligned}
& U^{(n+1)} \equiv \mathbf{u}_{n+1}-\mathbf{u}_{n}, \\
& Q^{(n+1)} \equiv q_{n+1}-q_{n}, \quad R^{(n+1)} \equiv r_{n+1}^{s}-r_{n}^{s} .
\end{aligned}
$$

Subtracting (3) with index $n$ from that with index $n+1$, we have

$$
\begin{gathered}
\frac{\partial U^{(n+1)}}{\partial t}-\nu \nabla_{u_{n}}^{2} U^{(n+1)}+\nabla_{u_{n}} Q^{(n+1)}=\mathscr{F}_{1}^{(n)}, \\
\nabla_{u_{n}} \cdot U^{(n+1)}=\mathscr{F}_{2}^{(n)} \quad \text { in } \Omega_{0 T}, \\
\nu \Pi \Pi_{u_{n}} \mathbf{D}_{u_{n}}\left(U^{(n+1)}\right) \mathbf{n}_{u_{n}}+\gamma \Pi \nabla_{\Gamma_{n, t}} R^{(n+1)}=\mathscr{F}_{3}^{(n)}, \\
\mathbf{n} \cdot \mathbf{T}_{u_{n}}\left(U^{(n+1)}, Q^{(n+1)}\right) \mathbf{n}_{u_{n}}-\bar{\Theta} \mathbf{n} \cdot \nabla_{\Gamma_{n, t}}^{2} \int_{0}^{t} U^{(n+1)} d \tau \\
+\gamma \mathbf{n} \cdot \nabla_{\Gamma_{n, t}} R^{(n+1)} \\
=b^{(n)}+\int_{0}^{t} \mathscr{B}^{(n)} d \tau \quad \text { on } \Gamma_{0 T},
\end{gathered}
$$




$$
\begin{gathered}
\left.U^{(n+1)}\right|_{t=0}=\mathbf{0} \quad \text { on } \bar{\Omega} \\
\frac{\partial R^{(n+1)}}{\partial t}-\chi \gamma \rho_{0}^{s} \nabla_{\Gamma_{n, t}}^{2} R^{(n+1)}=\mathscr{G}^{(n)} \quad \text { in } \Gamma_{0 T} \\
\left.R^{(n+1)}\right|_{t=0}=0 \quad \text { on } \Gamma,
\end{gathered}
$$

where

$$
\begin{gathered}
\mathscr{F}_{1}^{(n)} \equiv \nu\left(\nabla_{u_{n}}^{2}-\nabla_{u_{n-1}}^{2}\right) \mathbf{u}_{n}-\left(\nabla_{u_{n}}-\nabla_{u_{n-1}}\right) q_{n}, \\
\mathscr{F}_{2}^{(n)} \equiv-\left(\nabla_{u_{n}}-\nabla_{u_{n-1}}\right) \cdot \mathbf{u}_{n}, \\
\mathscr{F}_{3}^{(n)} \equiv-\nu \prod\left\{\Pi_{u_{n}} \mathbf{D}_{u_{n}}\left(\mathbf{u}_{n}\right) \mathbf{n}_{u_{n}}-\Pi_{u_{n-1}} \mathbf{D}_{u_{n-1}}\left(\mathbf{u}_{n}\right) \mathbf{n}_{u_{n-1}}\right\} \\
-\gamma \Pi\left(\nabla_{\Gamma_{n, t}}-\nabla_{\Gamma_{n-1, t}}\right) r_{n}^{s}, \\
b^{(n)} \equiv-\mathbf{n} \cdot\left\{\mathbf{T}_{u_{n}}\left(\mathbf{u}_{n}, q_{n}\right) \mathbf{n}_{u_{n}}-\mathbf{T}_{u_{n-1}}\left(\mathbf{u}_{n}, q_{n}\right) \mathbf{n}_{u_{n-1}}\right\} \\
-\gamma \mathbf{n} \cdot\left(\nabla_{\Gamma_{n, t}}-\nabla_{\Gamma_{n-1, t}}\right) r_{n}^{s}+b\left(\mathbf{u}_{n}, r_{n}^{s}\right)-b\left(\mathbf{u}_{n-1}, r_{n-1}^{s}\right) \\
\mathscr{B}^{(n)} \equiv \bar{\Theta} \mathbf{n} \cdot\left(\nabla_{\Gamma_{n, \tau}}^{2}-\nabla_{\Gamma_{n-1, \tau}}^{2}\right) \int_{0}^{\tau} \mathbf{u}_{n}(\xi, s) d s \\
+\bar{\Theta} \mathbf{n} \cdot\left(\nabla_{\Gamma_{n, \tau}}^{2}-\nabla_{\Gamma_{n-1, \tau}}^{2}\right) \mathbf{u}_{n}+B\left(\mathbf{u}_{n}, r_{n}^{s}\right)-B\left(\mathbf{u}_{n-1}, r_{n-1}^{s}\right) \\
\mathscr{G}^{(n)} \equiv \chi \gamma \rho_{0}^{s}\left(\nabla_{\Gamma_{n, t}}^{2}-\nabla_{\Gamma_{n-1, t}}^{2}\right) r_{n}^{s}+G\left(\mathbf{u}_{n}, r_{n}^{s}\right)-G\left(\mathbf{u}_{n-1}, r_{n-1}^{s}\right)
\end{gathered}
$$

Now, noting that the relations $\sum_{j=1}^{3} \partial_{\xi_{j}} A_{i j}=0$ hold for the cofactors $A_{i j}$ of the Jacobian matrix of any transformation from $\xi$ to $x$, by direct calculations, we can verify that the following relations hold:

$$
\frac{\partial \mathscr{F}_{2}^{(n)}}{\partial t}-\nabla_{u_{n}} \cdot \mathscr{F}_{1}^{(n)}=\nabla \cdot \mathbf{h}^{(n)}, \quad \mathbf{h}^{(n)}=\sum_{k=1}^{3} \partial_{\xi_{k}} \mathbf{H}_{k}^{(n)}
$$

where

$$
\begin{aligned}
\mathbf{H}_{k}^{(n)}= & -\left(\mathscr{J}_{u_{n}}^{-1}-\mathscr{J}_{u_{n-1}}^{-1}\right) \mathbf{L}_{k}^{(n)}-\mathscr{J}_{u_{n}}^{-1} \mathbf{M}_{k}^{(n)} \\
& +\frac{1}{4 \pi} \partial_{\xi_{k}} \int_{\Omega} \frac{\mathbf{N}_{k}^{(n)}(\eta, t)}{|\xi-\eta|} d \eta
\end{aligned}
$$

with

$$
\begin{aligned}
\mathbf{L}_{k}^{(n)} \equiv & \nu A_{i k}^{(n-1)} A_{i l}^{(n-1)} \partial_{\xi_{l}} \mathbf{u}_{n}-\mathscr{J}_{u_{n-1}}^{*} \mathbf{e}_{k} q_{n}, \\
\mathbf{M}_{k}^{(n)} \equiv & \nu\left(A_{i k}^{(n)} A_{i l}^{(n)}-A_{i k}^{(n-1)} A_{i l}^{(n-1)}\right) \partial_{\xi_{l}} \mathbf{u}_{n} \\
& -\left(\mathcal{J}_{u_{n}}^{*}-\mathcal{J}_{u_{n-1}}^{*}\right) \mathbf{e}_{k} q_{n}, \\
\mathbf{N}_{k}^{(n)} \equiv & \left\{\partial_{t}\left(\mathscr{J}_{u_{n}}^{-1}-\mathscr{J}_{u_{n-1}}^{-1}\right)\right\} \mathbf{u}_{n} \\
& -\left\{\partial_{\eta_{k}}\left(\mathcal{F}_{u_{n}}^{-1}-\mathcal{J}_{u_{n-1}}^{-1}\right)\right\} \mathbf{L}_{k}^{(n)}-\left(\partial_{\eta_{k}} \mathscr{J}_{u_{n}}^{-1}\right) \mathbf{M}_{k}^{(n)} .
\end{aligned}
$$

In (66), the Einstein summation convention is used, $A_{i j}^{(n)}$ denotes the $(i, j)$-component of $\mathscr{J}_{n}^{*}$, and $\mathbf{e}_{k}, k=1,2,3$, denote fundamental unit vectors in $\mathbf{R}^{3}$.

For the terms in (63), $\mathbf{h}^{(n)}$, and $\mathbf{H}_{k}^{(n)}$, we have the following estimate for arbitrary $0<\epsilon<1$ :

$$
\begin{gathered}
\left|\mathscr{F}_{1}^{(n)}\right|_{\Omega_{0 T}}^{(\alpha,(\alpha / 2))}+\left|\mathscr{F}_{2}^{(n)}\right|_{\Omega_{0 T}}^{(1+\alpha,((1+\alpha) / 2))}+\left|\mathscr{F}_{3}^{(n)}\right|_{\Gamma_{0 T}}^{(1+\alpha,((1+\alpha) / 2))} \\
+\left|b^{(n)}\right|_{\Gamma_{0 T}}^{(1+\alpha,((1+\alpha) / 2))}+\left|\mathscr{B}^{(n)}\right|_{\Gamma_{0 T}}^{(\alpha,(\alpha / 2))}+\left|\mathbf{h}^{(n)}\right|_{\Omega_{0 T}}^{(\alpha,(\alpha / 2))} \\
+\sum_{k=1}^{3}\left|\mathbf{H}_{k}^{(n)}\right|_{\Omega_{0 T}}^{(1+\alpha, \gamma)}+\left|\mathscr{G}^{(n)}\right|_{\Gamma_{0 T}}^{(\alpha,(\alpha / 2))} \\
\leq C_{1}\left\{\epsilon\left(\left|U^{(n)}\right|_{\Omega_{0 T}}^{(2+\alpha, 1+(\alpha / 2))}+\left|R^{(n)}\right|_{\Gamma_{0 T}}^{(2+\alpha, 1+(\alpha / 2))}\right)\right. \\
+C(\epsilon) \int_{0}^{T}\left(\left|U^{(n)}\right|_{\Omega_{0 \tau}}^{(2+\alpha, 1+(\alpha / 2))}\right. \\
\left.\left.+\left|R^{(n)}\right|_{\Gamma_{0 \tau}}^{(2+\alpha, 1+(\alpha / 2))}\right) d \tau\right\}
\end{gathered}
$$

We will derive here the estimate only of $\mathbf{H}_{k}^{(n)}$ because the other terms can be estimated in a similar and simpler manner. Noting that the following estimates hold for $\mathbf{u}_{n}$ and $q_{n}$ :

$$
\left|\mathbf{u}_{n}\right|_{\Omega_{0 T}}^{(2+\alpha, 1+(\alpha / 2))}+\left|q_{n}\right|_{\Omega_{0 T}}^{(1+\alpha, \gamma)} \leq C_{2},
$$

and with the aid of estimates (38) and (39), we have

$$
\begin{gathered}
\left|\left(\mathscr{J}_{u_{n}}^{-1}-\mathscr{J}_{u_{n-1}}^{-1}\right) \mathbf{L}_{k}^{(n)}\right|_{\Omega_{0 T}}^{(1+\alpha, \gamma)},\left|\mathscr{J}_{u_{n}}^{-1} \mathbf{M}_{k}^{(n)}\right|_{\Omega_{0 T}}^{(1+\alpha, \gamma)},\left|\mathbf{N}_{k}^{(n)}\right|_{\Omega_{0 T}}^{(0,((1+\alpha) / 2))} \\
\leq C_{3}\left(\epsilon\left|U^{(n)}\right|_{\Omega_{0 T}}^{(2+\alpha, 1+(\alpha / 2))}\right. \\
\left.+C(\epsilon) \int_{0}^{T}\left|U^{(n)}\right|_{\Omega_{0 \tau}}^{(2+\alpha, 1+(\alpha / 2))} d \tau\right) .
\end{gathered}
$$

Noting also the following inequality:

$$
\left|\partial_{\xi_{k}} \int_{\Omega} \frac{\mathbf{N}_{k}^{(n)}(\eta, t)}{|\xi-\eta|} d \eta\right|_{\Omega_{0 T}}^{(1+\alpha, \gamma)} \leq C_{4}\left|\mathbf{N}_{k}^{(n)}\right|_{\Omega_{0 T}}^{(0,((1+\alpha-\gamma) / 2))}
$$

from estimate (69) for $\mathbf{N}_{k}^{(n)}$, we have

$$
\begin{aligned}
&\left|\partial_{\xi_{k}} \int_{\Omega} \frac{\mathbf{N}_{k}^{(n)}(\eta, t)}{|\xi-\eta|} d \eta\right|_{\Omega_{0 T}}^{(1+\alpha, \gamma)} \\
& \leq C_{5}\left(\epsilon\left|U^{(n)}\right|_{\Omega_{0 T}}^{(2+\alpha, 1+(\alpha / 2))}\right. \\
&\left.\quad+C(\epsilon) \int_{0}^{T}\left|U^{(n)}\right|_{\Omega_{0 \tau}}^{(2+\alpha, 1+(\alpha / 2))} d \tau\right) .
\end{aligned}
$$

Thus, from estimates (69) and (71), we have the desired estimate for $\mathbf{H}_{k}^{(n)}$. 
Now, applying Theorem 4 to problem (62), we have the following estimate:

$$
\begin{aligned}
\left|U^{(n+1)}\right|_{\Omega_{0 T}}^{(2+\alpha, 1+(\alpha / 2))}+\left\|Q^{(n+1)}\right\|_{T}+\left|R^{(n+1)}\right|_{\Gamma_{0 T}}^{(2+\alpha, 1+(\alpha / 2))} \\
\leq C_{6}\left\{\epsilon\left(\left|U^{(n)}\right|_{\Omega_{0 T}}^{(2+\alpha, 1+(\alpha / 2))}+\left|R^{(n)}\right|_{\Gamma_{0 T}}^{(2+\alpha, 1+(\alpha / 2))}\right)\right. \\
\left.+C(\epsilon) \int_{0}^{T}\left(\left|U^{(n)}\right|_{\Omega_{0 \tau}}^{(2+\alpha, 1+(\alpha / 2))}+\left|R^{(n)}\right|_{\Gamma_{0 \tau}}^{(2+\alpha, 1+(\alpha / 2))}\right) d \tau\right\},
\end{aligned}
$$

where the norm $\|f\|_{T}$ is defined as $\|f\|_{T} \equiv|f|_{\Omega_{0 T}}^{(1+\alpha, \gamma)}+$ $|\nabla f|_{\Omega_{0 T}}^{(\alpha,(\alpha / 2))}+|f|_{\Gamma_{0 T}}^{(1+\alpha,((1+\alpha) / 2))}$. Fixing $\epsilon$ so that $C_{6} \epsilon \leq 1 / 2$ and summing the above inequalities from $n=1$ to $n=m$, we have

$$
S_{m}(T) \leq C_{7}\left(S_{1}(T)+\int_{0}^{T} S_{m}(\tau) d \tau\right)
$$

where

$$
S_{m}(T) \equiv \sum_{n=1}^{m}\left(\left|U^{(n)}\right|_{\Omega_{0 T}}^{(2+\alpha, 1+(\alpha / 2))}+\left\|Q^{(n)}\right\|_{T}+\left|R^{(n)}\right|_{\Gamma_{0 T}}^{(\alpha,(\alpha / 2))}\right) .
$$

Then, using Gronwall's inequality, from the above inequality, we have

$$
S_{m}(T) \leq C_{8}\left(1+T e^{C_{9} T}\right) .
$$

Noting that the right-hand side in (75) is independent of $m$, we can conclude that the sequence $\left\{\left(\mathbf{u}_{n}, q_{n}, r_{n}^{s}\right)\right\}$ is convergent in $C^{2+\alpha, 1+(\alpha / 2)}\left(\Omega_{0 T}\right) \times\left(C^{1+\alpha, \gamma}\left(\Omega_{0 T}\right) \cap C^{1+\alpha,((1+\alpha) / 2)}\left(\Gamma_{0 T}\right)\right) \times$ $C^{2+\alpha, 1+(\alpha / 2)}\left(\Gamma_{0 T}\right)$.

Now, let us prove Theorem 1 . Taking the limit as $n$ tends to infinity in problem (3), we can easily see that the limit of the sequence $\left\{\left(\mathbf{u}_{n}, q_{n}, r_{n}^{s}\right)\right\}$ is a solution of problem (11)-(13). The uniqueness can be proved as follows. Let $\left(\mathbf{u}, q, r^{s}\right)$ and $\left(\widetilde{\mathbf{u}}, \tilde{q}, \widetilde{r}^{s}\right)$ be two solutions of problem (11)-(13). By subtracting one equation from the other, we obtain the equations for the differences $V \equiv \mathbf{u}-\widetilde{\mathbf{u}}, P \equiv q-\widetilde{q}$, and $R \equiv r^{s}-\widetilde{r}^{s}$, the form of which is similar to (62). Then, in a similar manner to obtain (72), we can obtain the following estimate:

$$
\begin{aligned}
& |V|_{\Omega_{0 T}}^{(2+\alpha, 1+(\alpha / 2))}+|R|_{\Gamma_{0 T}}^{(2+\alpha, 1+(\alpha / 2))} \\
& \leq C_{10}\left\{\epsilon\left(|V|_{\Omega_{0 T}}^{(2+\alpha, 1+(\alpha / 2))}+|R|_{\Gamma_{0 T}}^{(2+\alpha, 1+(\alpha / 2))}\right)\right. \\
& \left.\quad+C(\epsilon) \int_{0}^{T}\left(|V|_{\Omega_{0 \tau}}^{(2+\alpha, 1+(\alpha / 2))}+|R|_{\Gamma_{0 \tau}}^{(2+\alpha, 1+(\alpha / 2))}\right) d \tau\right\} .
\end{aligned}
$$

This inequality implies that $V=\mathbf{0}$ and $R=0$, and as a consequence, $P=0$ follows. Thus, the proof of Theorem 1 is completed.

\section{References}

[1] Y. D. Shikhmurzaev, Capillary Flows with Forming Interfaces, Chapman \& Hall/CRC, Boca Raton, Fla, USA, 2008.
[2] Y. D. Shikhmurzaev, "The moving contact line on a smooth solid surface," International Journal of Multiphase Flow, vol. 19, no. 4, pp. 589-610, 1993.

[3] Y. D. Shikhmurzaev, "Moving contact lines in liquid/liquid/ solid systems," Journal of Fluid Mechanics, vol. 334, pp. 211-249, 1997.

[4] Y. D. Shikhmurzaev, "On cusped interfaces," Journal of Fluid Mechanics, vol. 359, pp. 313-328, 1998.

[5] H. Wong, D. Rumschitzki, and C. Maldarelli, "On the surfactant mass balance at a deforming fluid interface," Physics of Fluids, vol. 8, no. 11, pp. 3203-3204, 1996.

[6] I. Sh. Mogilevskiĭ and V. A. Solonnikov, "On the solvability of an evolution free boundary problem for the Navier-Stokes equations in Hölder spaces of functions," in Mathematical Problems Relating to the Navier-Stokes Equation, vol. 11 of Advances in Mathematics for Applied Sciences, pp. 105-181, World Science Publications, River Edge, NJ, USA, 1992.

[7] O. A. Lady ženskaja, V. A. Solonnikov, and N. N. Ural'ceva, "Linear and quasi-linear equations of parabolic type," Translations of Mathematical Monographs, 1968. 


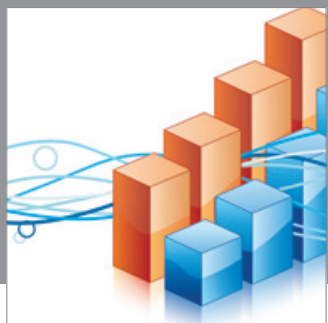

Advances in

Operations Research

mansans

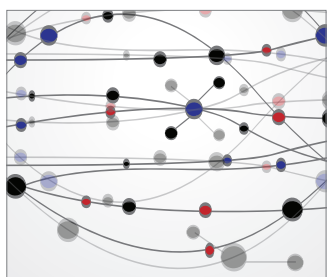

The Scientific World Journal
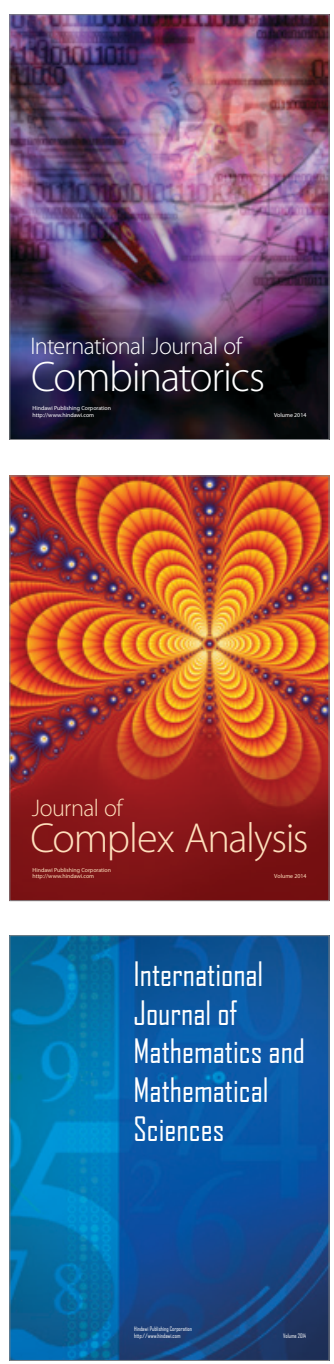
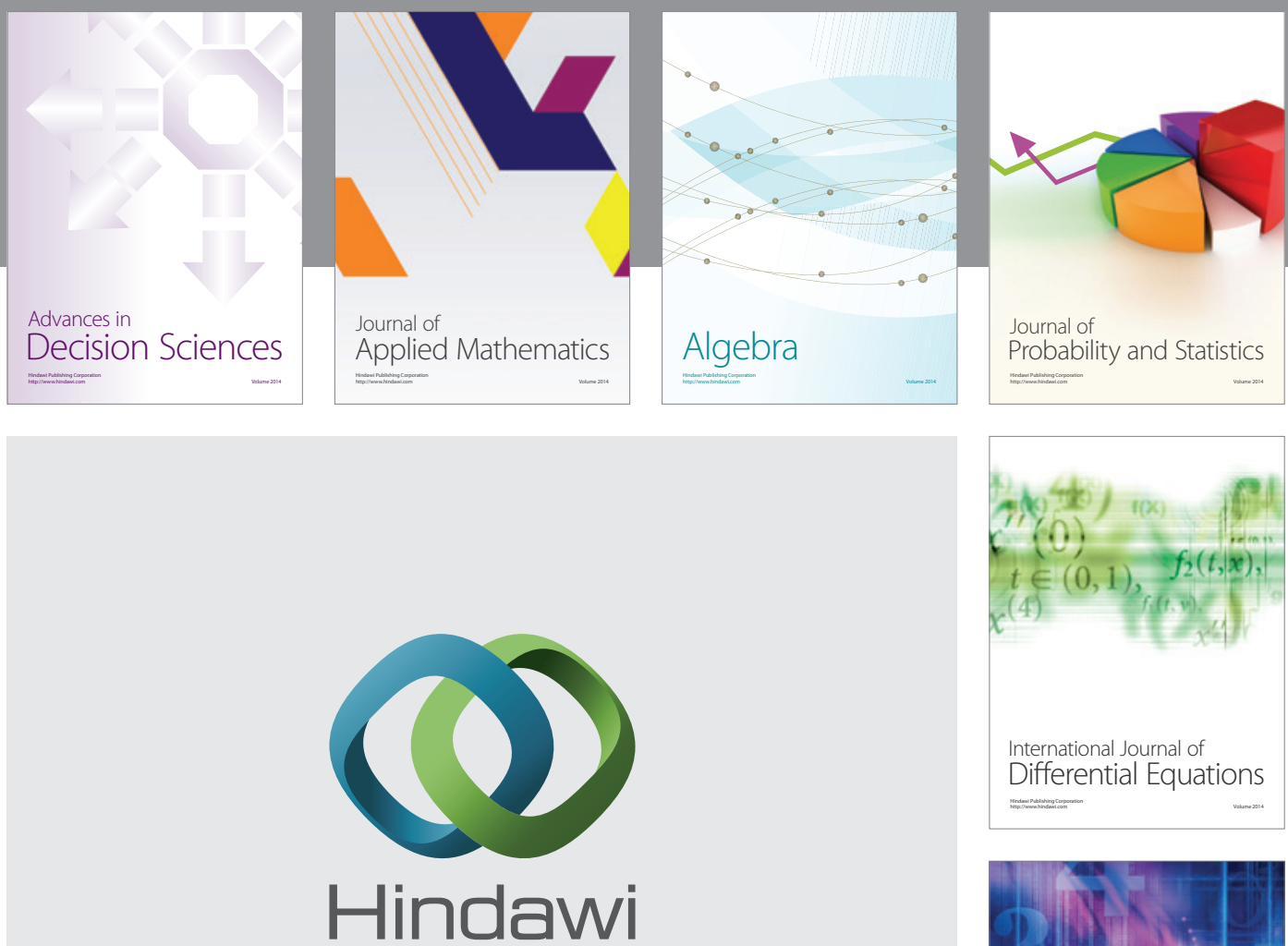

Submit your manuscripts at http://www.hindawi.com
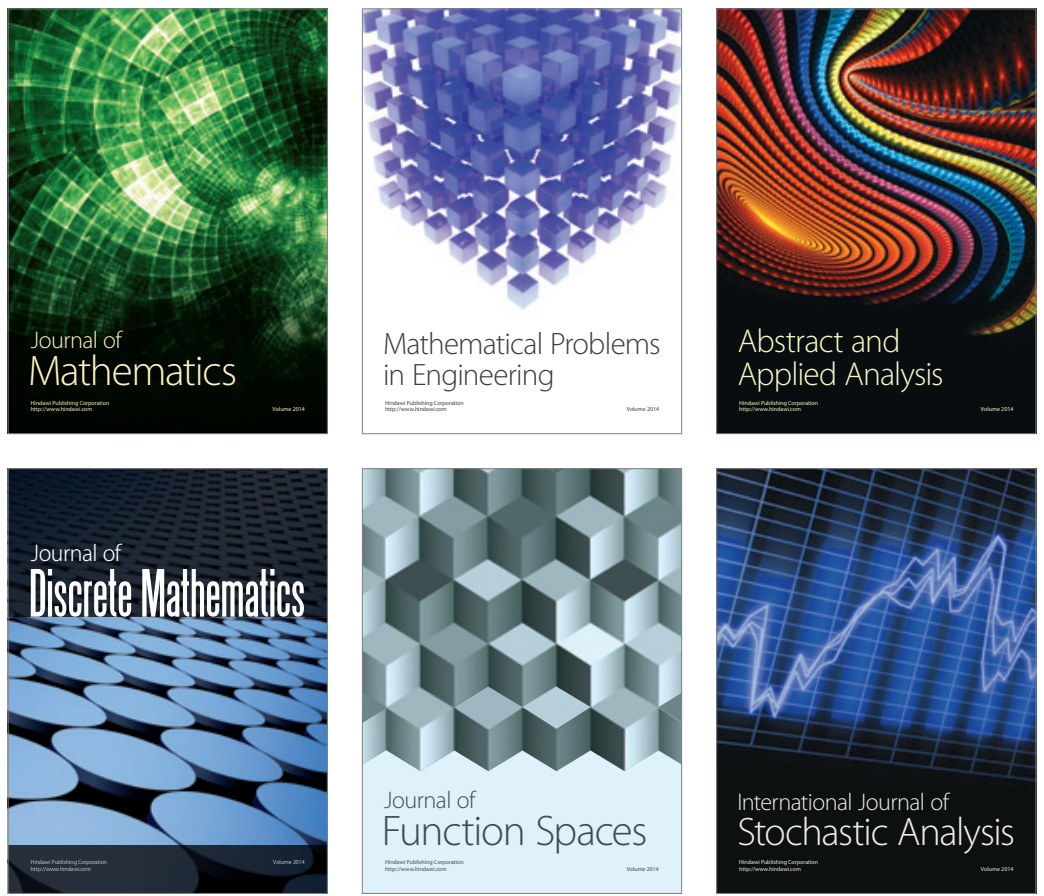

Journal of

Function Spaces

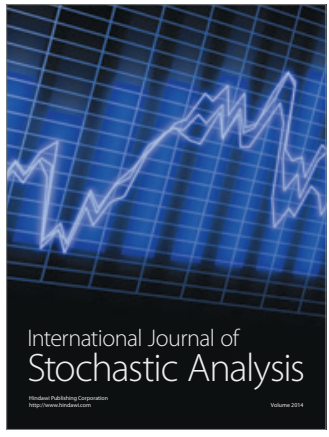

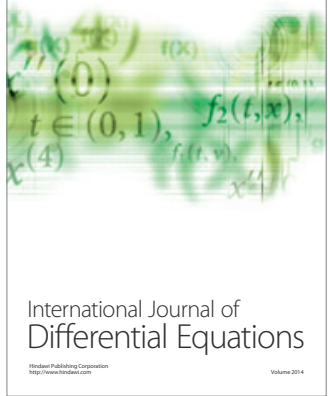
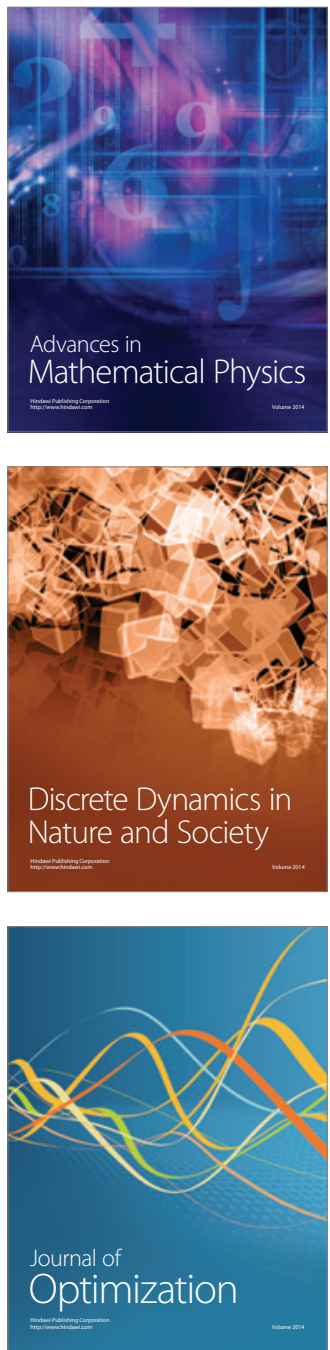